\title{
Syphilis: Cutting risk through primary prevention and prenatal screening
}

\author{
This highly infectious STI poses severe consequences to women and babies \\ when infection occurs during pregnancy, with infection rates higher among \\ women who lack prenatal care. These authors stress education of at-risk \\ populations and early recognition of clinical features to quell rising infection rates.
}

Tory A. Finley, BA, and Patrick Duff, MD

\section{IN THIS ARTICLE}

Today's syphilis problem

page 22

Prenatal screening and diagnosis

page 24

\section{Treatment in}

pregnancy

page 26
CASE Pregnant woman with positive Treponema pallidum antibody test

A 30-year-old primigravida at 10 weeks and 4 days of gestation by her last menstrual period presents to your office for her initial prenatal visit. She expresses no concerns. You order the standard set of laboratory tests, including a sexually transmitted infection (STI) screening panel. Consistent with your institution's use of the reverse algorithm for syphilis screening, you obtain a Treponema pallidum antibody test, which reflexes to the rapid plasma reagin (RPR) test. Three days later, you receive a notification that this patient's $T$ pallidum antibody result was positive, followed by negative RPR test results. The follow-up $T$ pallidum particle agglutination (TP-PA) test also was negative. Given these findings, you consider:

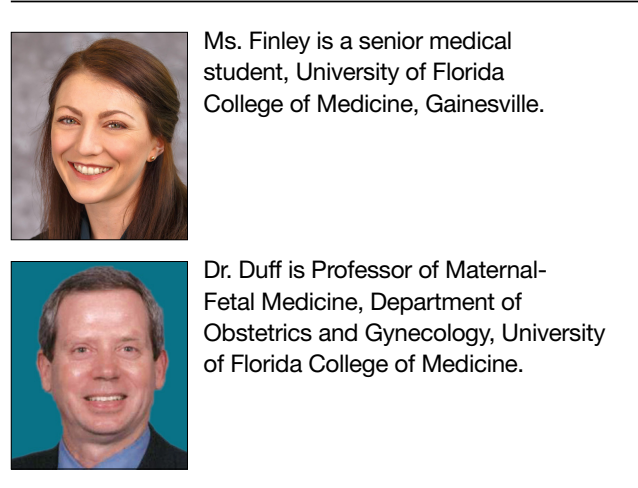

The authors report no financial relationships relevant to this article. doi: 10.12788/obgm.0047
- What is the correct interpretation of the patient's sequence of test results?

- Is she infected, and does she require treatment?

\section{Meet our perpetrator}

Syphilis has plagued society since the late 15th century, although its causative agent, the spirochete $T$ pallidum, was not recognized until 1905. ${ }^{1,2} T$ pallidum bacteria are transmitted via sexual contact, as well as through vertical transmission during pregnancy or delivery. Infection with syphilis is reported in $50 \%$ to $60 \%$ of sexual partners after a single exposure to an infected individual with early syphilis, and the mean incubation period is 21 days. $^{3} T$ pallidum can cross the placenta and infect a fetus as early as the sixth week of gestation. ${ }^{3}$ Congenital syphilis infections occur in the neonates of $50 \%$ to $80 \%$ of women with untreated primary, secondary, or early latent syphilis infections; maternal syphilis is associated with a $21 \%$ increased risk of stillbirth, a $6 \%$ increased risk of preterm delivery, and a $9 \%$ increased risk of neonatal death. ${ }^{4,5}$ Additionally, syphilis infection is associated with a high risk of HIV infection, as well as coinfection with other STIs. ${ }^{1}$

Given the highly infective nature of $T$ pallidum, as well as the severity of the potential consequences of infection for both mothers and babies, primary prevention, 
FIGURE 1 The chancre that is characteristic of primary syphilis

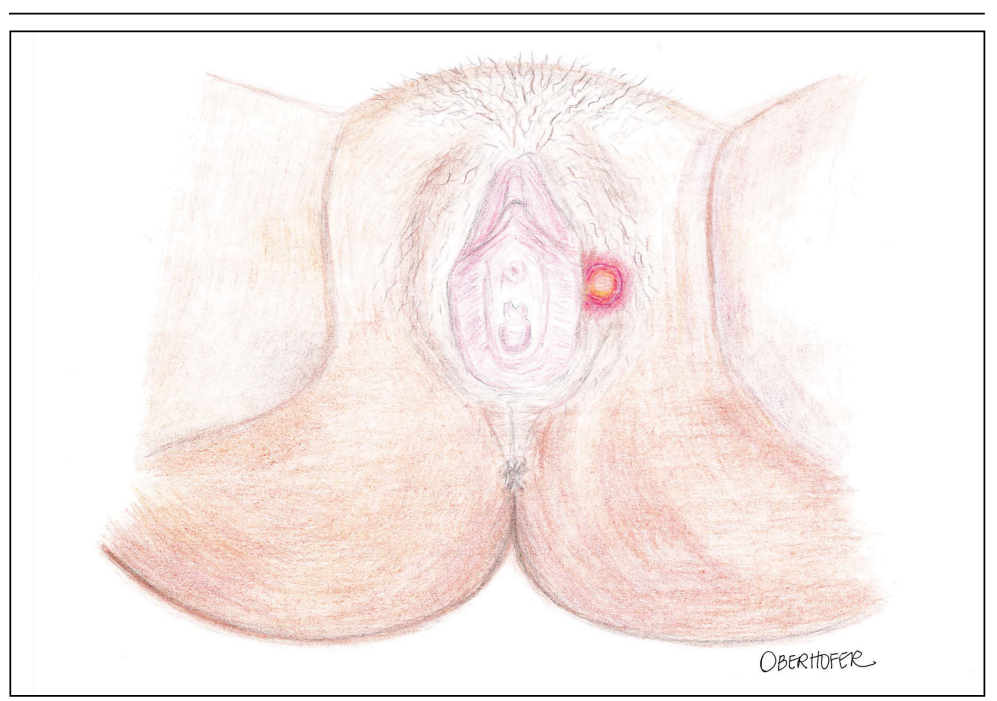

Image courtesy of Haley Oberhofer.

\section{FIGURE 2 The characteristic rash of secondary syphilis}

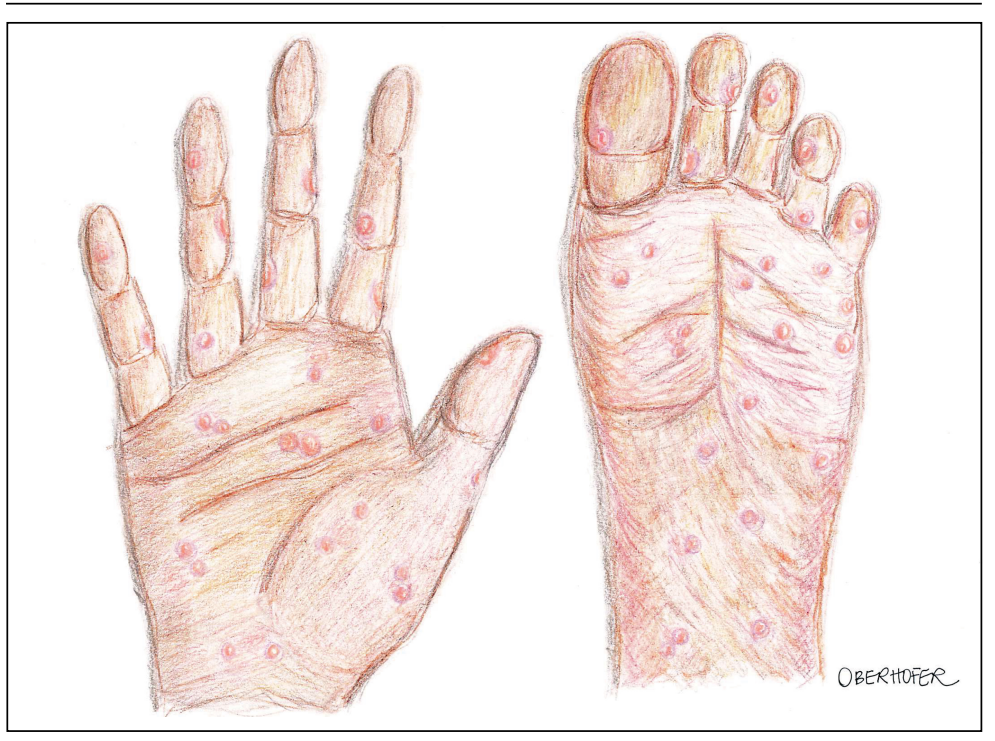

Image courtesy of Haley Oberhofer.

education of at-risk populations, and early recognition of clinical features of syphilis infection are of utmost importance in preventing morbidity and mortality. In this article, we review the epidemiology and extensive clinical manifestations of syphilis, as well as current screening recommendations and treatment for pregnant women.

\section{The extent of the problem today}

Although US rates of syphilis have ebbed and flowed for the past several decades, the current incidence has grown exponentially in recent years, with the number of cases reported to the Centers for Disease Control and Prevention (CDC) increasing by $71 \%$ from 2014 to $2018 .{ }^{6}$ During this time period, reported cases of primary and secondary syphilis in women more than doubled (172.7\% and $165.4 \%$, respectively) according to CDC data, accompanied by a parallel rise in reported cases of congenital syphilis in both live and stillborn infants. ${ }^{6}$ In 2018, the CDC reported a national rate of congenital syphilis of 33.1 cases per 100,000 live births, a $39.7 \%$ rise compared with data from $2017 .^{6}$ Those most at risk. Risk factors for syphilis infection include age younger than 30 years, low socioeconomic status, substance abuse, HIV infection, concurrent STIs, and high-risk sexual activity (sex with multiple high-risk partners). ${ }^{3}$ Additionally, reported rates of primary and secondary syphilis infections, as well as congenital syphilis infections, are more elevated among women who identify as Black, American Indian/Alaska Native, and/or Hispanic. ${ }^{6}$ Congenital infections in the United States are correlated with a lack of prenatal care, which has been similarly linked with racial and socioeconomic disparities, as well as with untreated mental health and substance use disorders and recent immigration to the United States. ${ }^{5,7}$

\section{The many phases of syphilis}

The characteristic lesion of primary syphilis is a chancre, which is a painless, ulcerative lesion with raised borders and a clean, indurated base appearing at the site of spirochete entry (FIGURE 1). Chancres most commonly appear in the genital area, with the most frequent sites in females being within the vaginal canal or on the cervix. Primary chancres tend to heal spontaneously within 3 to 6 weeks, even without treatment, and frequently are accompanied by painless inguinal lymphadenopathy. Given that the most common chancre sites are not immediately 
apparent, primary infections in women often go undetected. ${ }^{3}$ In fact, it is essential for clinicians to recognize that, in our routine practice, most patients with syphilis will not be symptomatic at all, and the diagnosis will only be made by serologic screening.

Following resolution of the primary phase, the patient may enter the secondary stage of $T$ pallidum infection. During this stage, spirochetes may disseminate throughout the bloodstream to infect all major organ systems. The principal manifestations of secondary syphilis include a diffuse maculopapular rash that begins on the trunk and proximal extremities and spreads to include the palms and soles (FIGURE 2); mucosal lesions, such as mucous patches and condyloma lata (FIGURE 3); nonscarring alopecia; periostitis; generalized lymphadenopathy; and, in some cases, hepatitis or nephritis. ${ }^{1,3}$

Secondary syphilis usually clears within 2 to 6 weeks, with the patient then entering the early latent stage of syphilis. During this period, up to $25 \%$ of patients are subject to flares of secondary syphilitic lesions but otherwise are asymptomatic. ${ }^{1,3,4}$ These recurrences tend to occur within 1 year, hence the distinction between early and late latent stages. Once a year has passed, patients are not contagious by sexual transmission and are unlikely to suffer a relapse of secondary symptoms. ${ }^{1,3}$ However, late latent syphilis is characterized by periods of intermittent bacteremia that allow for seeding of the placenta and infection in about $10 \%$ of fetuses. ${ }^{5}$

Untreated, about $40 \%$ of patients will progress to the tertiary stage of syphilis, which is characterized by gummas affecting the skin and mucous membranes (FIGURE 4) and cardiovascular manifestations including arterial aneurysms and aortic insufficiency. ${ }^{3}$

Neurologic manifestations of syphilis may arise during any of the above stages, though the most characteristic manifestations tend to appear decades after the primary infection. Early neurosyphilis may present as meningitis, with or without concomitant ocular syphilis (uveitis, retinitis) and/or as otic syphilis (hearing loss, persistent tinnitus). ${ }^{1,5}$ Patients with late (tertiary)

\section{FIGURE 3 Condyloma lata, which is characteristic of secondary syphilis}

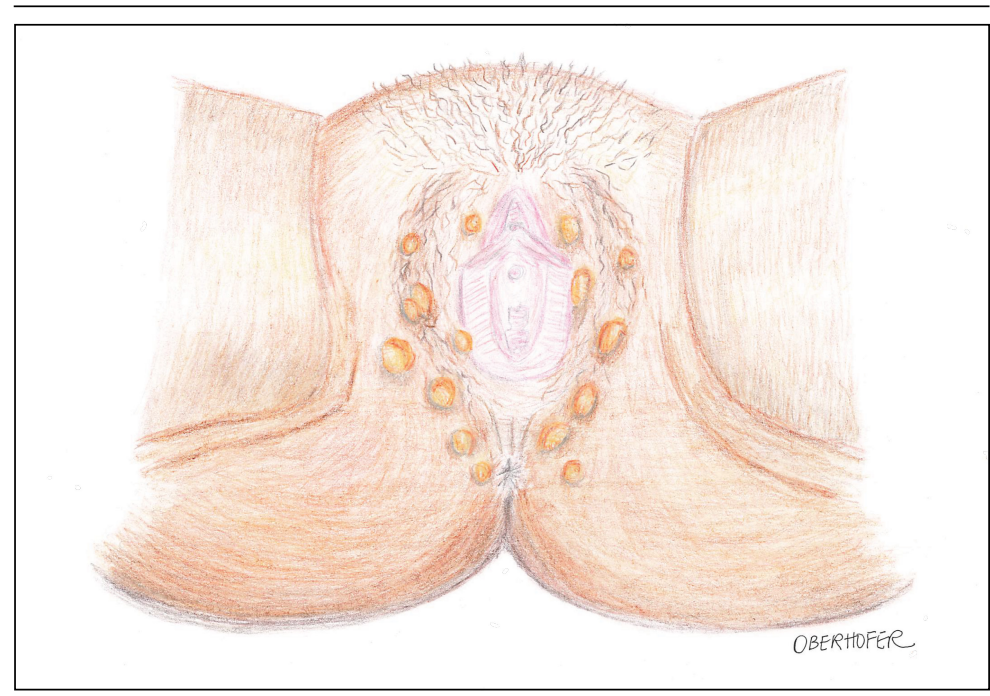

Image courtesy of Haley Oberhofer.

\section{FIGURE 4 The gumma, the characteristic mucocutaneous lesion of tertiary syphilis}

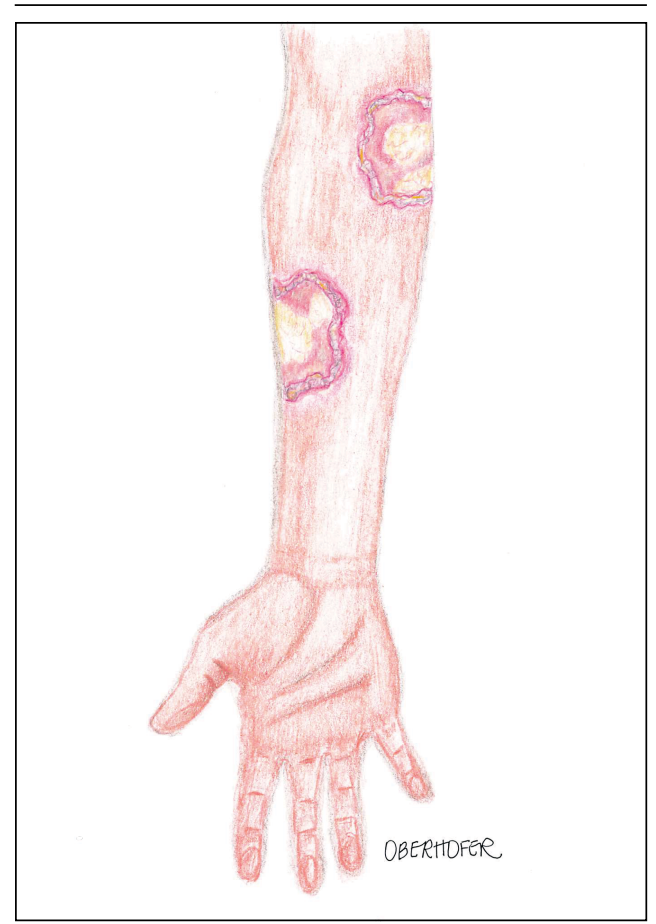

Image courtesy of Haley Oberhofer. 


\section{FIGURE 5 Traditional syphilis screening algorithm}

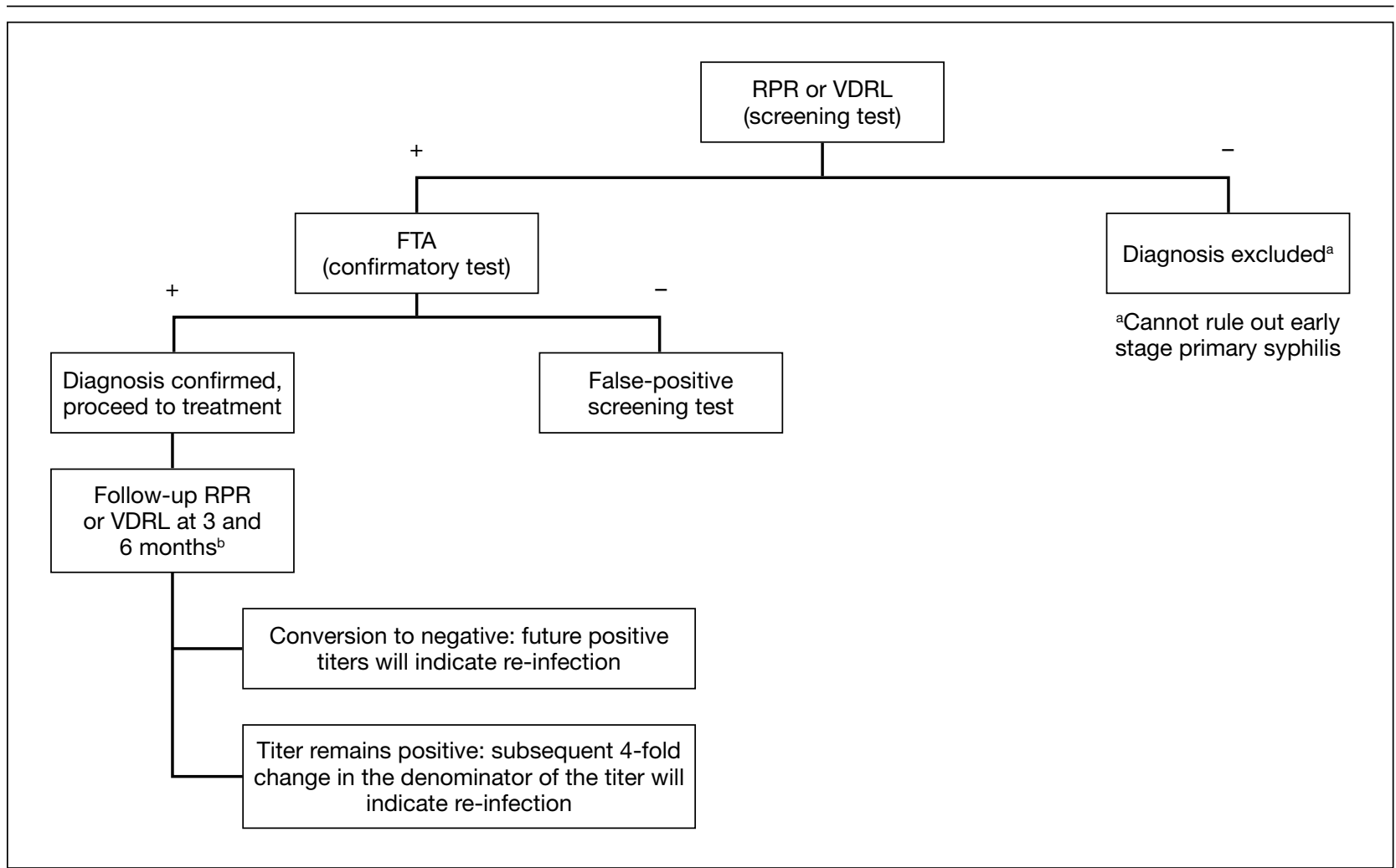

${ }^{\mathrm{b}}$ Follow-up titers should be ordered at 3 and 6 months to determine efficacy of treatment and to establish a baseline titer for future screening. A subsequent 4-fold increase in the denominator of this titer (ie, 1:8 $\rightarrow$ 1:32) indicates re-infection and a need for repeat treatment.

Abbreviations: FTA, fluorescent treponemal antibody; RPR, rapid plasma reagin; VDRL, Venereal Disease Research Laboratory.

neurosyphilis tend to exhibit meningovascular symptoms similar to stroke (aphasia, hemiplegia, seizures) and/or parenchymal effects such as general paresis. Tabes dorsalis (manifestations of which include urinary and rectal incontinence, lightning pains, and ataxia) is a late-onset manifestation. ${ }^{1,3}$

Congenital syphilis can be subdivided into an early and late stage. The first stage, in which clinical findings occur within the first 2 years of life, commonly features a desquamating rash, hepatomegaly, and rhinitis. Anemia, thrombocytopenia, periostitis, and osteomyelitis also have been documented. ${ }^{5}$ Of note, two-thirds of infants are asymptomatic at birth and may not develop such clinical manifestations for 3 to 8 weeks. ${ }^{3}$ If untreated, early congenital infection may progress to late manifestations, such as Hutchinson teeth, mulberry molars, interstitial keratitis, deafness, saddle nose, saber shins, and such neurologic abnormalities as developmental delay and general paresis. ${ }^{3}$

\section{Prenatal screening and diagnosis}

Current recommendations issued by the CDC and the American College of Obstetricians and Gynecologists state that all pregnant women should be screened for syphilis infection at their first presentation to care, with repeat screening between 28 and 32 weeks of gestation and at birth, for women living in areas with a high prevalence of syphilis and/or with any of the aforementioned risk factors. ${ }^{3,5}$ Given that providers may be unfamiliar with the prevalence of syphilis 


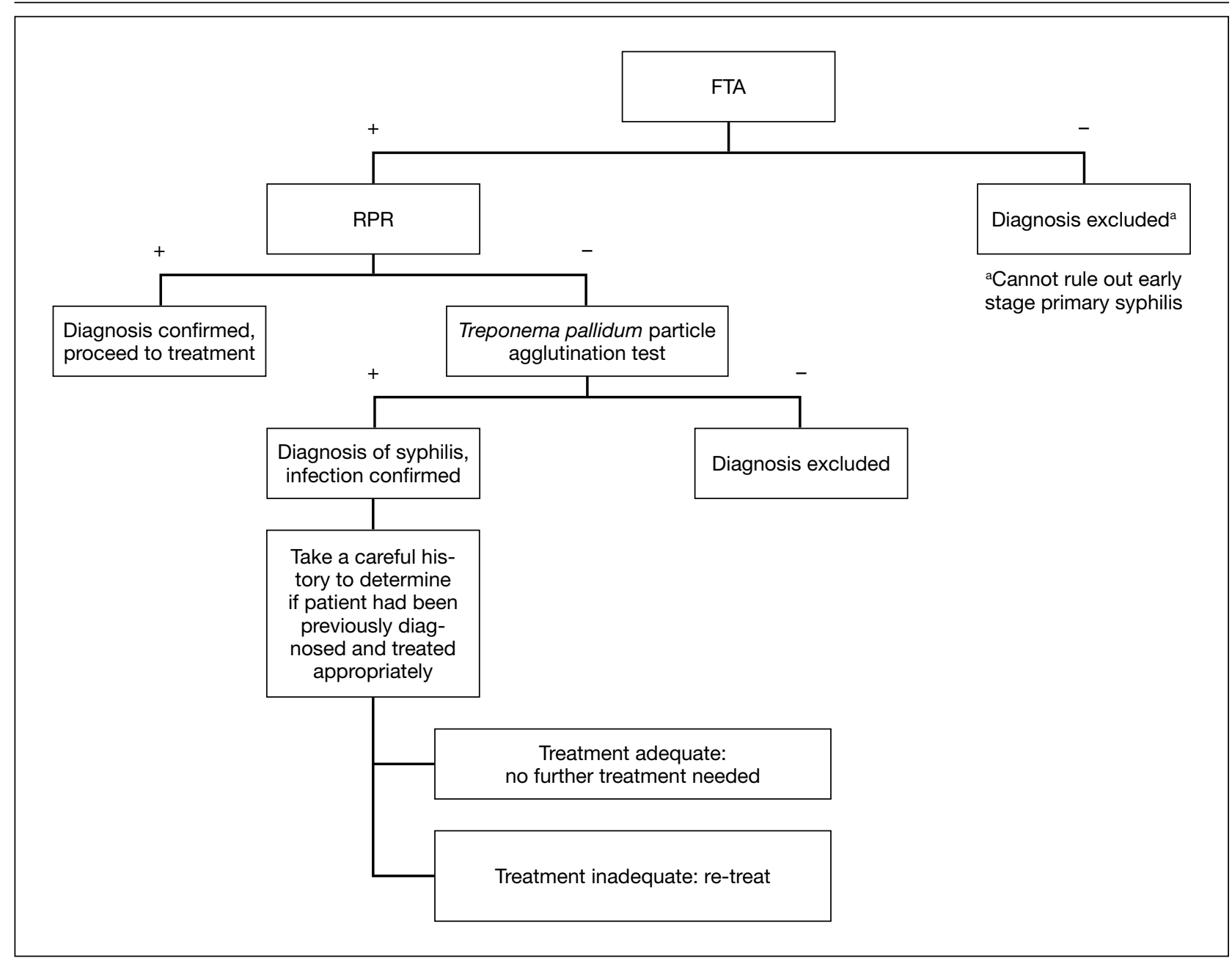

Abbreviations: FTA, fluorescent treponemal antibody; RPR, rapid plasma reagin.

in their area, and that patients may acquire or develop an infection later on in their pregnancy, researchers have begun to investigate the feasibility of universal third-trimester screening. While the cost-effectiveness of such a protocol is disputed, recent studies suggest that it may result in a substantial decrease in adverse maternal and fetal outcomes. ${ }^{8,9}$

\section{Diagnostic tests}

The traditional algorithm for the diagnosis of syphilis infection begins with a nontreponemal screening test, such as the RPR or the Venereal Disease Research Laboratory test. If positive, these screening tests are followed by a confirmatory treponemal test, such as the fluorescent treponemal antibody (FTA) test or the TP-PA (FIGURE 5).

The "reverse" screening algorithm begins with the FTA and, if positive, reflexes to the RPR. A reactive RPR indicates an active infection, and the patient should be treated. A negative RPR should be followed by the TP-PA to rule out a false-positive immunoglobulin G test. If the TP-PA test result is positive, the diagnosis of syphilis is confirmed (FIGURE 6). It is crucial to understand, however, that treponemal antibodies will remain positive for a patient's lifetime, and someone who may have been treated for syphilis in the past also will screen positive. Once 2 
TABLE Common presentations and treatment of syphilis in pregnancy

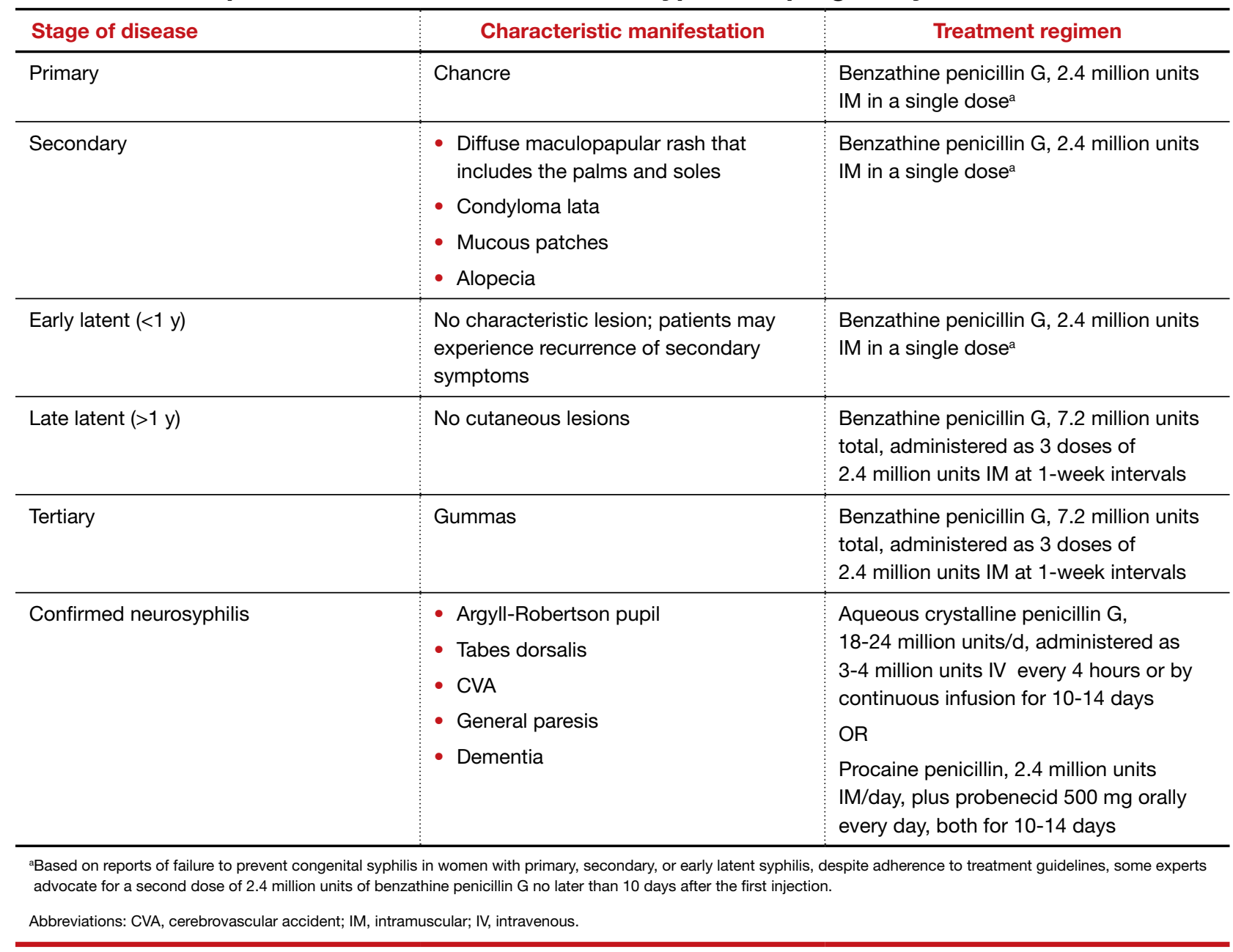

treponemal tests are positive, physicians should take a careful history to assess prior infection risk and treatment status. A negative TP-PA excludes a diagnosis of syphilis.

Advantages of the reverse screening algorithm. Nontreponemal tests are inexpensive and easy to perform, and titers allow for identification of a baseline to evaluate response to treatment. ${ }^{11}$ However, given the fluctuation of RPR sensitivity (depending on stage of disease and a decreased ability to detect primary and latent stages of syphilis), there has been a resurgence of interest in the reverse algorithm. ${ }^{11}$ While reverse screening has been found to incur higher costs, and may result in overtreatment and increased stress due to false-positive results, ${ }^{12}$ there is evidence to suggest that this algorithm is more sensitive for primary and latent infections. ${ }^{8,11,13-15}$

Given the rise in prevalence of syphilis infections in the United States over the past decade, and therefore a higher pretest probability of syphilis in the population, we favor the reverse screening algorithm in obstetrics, particularly given the risks of adverse maternal and fetal outcomes.

\section{Treating syphilis in pregnancy}

Parenteral benzathine penicillin $G$ is the only currently recommended medication for the treatment of syphilis in pregnancy. This drug is effective in treating maternal infection and in preventing fetal infections, as well as in 
treating established fetal infections., ${ }^{3,5}$ Regimens differ depending on the stage of syphilis infection (TABLE). Treatment for presumed early syphilis is recommended for women who have had sexual contact with a partner diagnosed with primary, secondary, or early latent syphilis within 3 months of their current pregnancy. ${ }^{5}$ Any patient with diagnosed syphilis who demonstrates clinical signs of neurologic involvement should undergo lumbar puncture to assess for evidence of neurosyphilis. ${ }^{3}$ CDC guidelines recommend that patients who report an allergy to penicillin undergo desensitization therapy in a controlled setting, as other antibiotics that have been investigated in the treatment of syphilis are either not appropriate due to teratogenicity or due to suboptimal fetal treatment.,

Syphilotherapy may lead to the JarischHerxheimer reaction, which is an acute systemic reaction to inflammatory cytokines produced in response to lipopolysaccharide released by dying spirochetes. ${ }^{5}$ This reaction is characterized by fever, chills, myalgia, headache, hypotension, and worsening of cutaneous lesions. Preterm labor and delivery and fetal heart rate tracing abnormalities also have been documented in pregnant women experiencing this reaction, particularly during the second half of pregnancy. ${ }^{16}$ Prior to the start of treatment, a detailed sonographic assessment should be performed to assess the fetus for signs of early syphilis, including hepatomegaly, elevated peak systolic velocity of the middle cerebral artery (indicative of fetal anemia), polyhydramnios, placentomegaly, or hydrops. ${ }^{5,7}$

\section{CASE Resolved}

The combination of the patient's test results positive FTA, negative RPR, and negative TP-PA-suggest a false-positive treponemal assay. This sequence of tests excludes a diagnosis of syphilis; therefore, no treatment is necessary. Depending on the prevalence of syphilis in the patient's geographic location, as well as her sexual history, rescreening between 28 and 32 weeks may be warranted.

\section{References}

1. Ghanem KG, Ram S, Rice PA. The modern epidemic of syphilis. N Engl J Med. 2020;382:845-854.

2. Barnett R. Syphilis. Lancet. 2018;391:1471.

3. Duff P. Maternal and fetal infections. In: Resnik R, Lockwood CJ, Moore T, et al. Creasy and Resnik's Maternal-Fetal Medicine: Principles and Practice. 8th ed. Philadelphia, PA: Elsevier; 2018:862-919.

4. Gomez GB, Kamb ML, Newman LM, et al. Untreated maternal syphilis and adverse outcomes of pregnancy: a systematic review and meta-analysis. Bull World Health Organ. 2013;91:217-226.

5. Adhikari EH. Syphilis in pregnancy. Obstet Gynecol. 2020;135:1121-1135.

6. Syphilis. CDC website. https://www.cdc.gov/std/stats18/syphilis .htm. Published October 1, 2019. Accessed October 6, 2020.

7. Rac MF, Revell PA, Eppes CS. Syphilis during pregnancy: a preventable threat to maternal-fetal health. Am J Obstet Gynecol. 2017;4:352-363.

8. Dunseth CD, Ford BA, Krasowski MD. Traditional versus reverse syphilis algorithms: a comparison at a large academic medical center. Pract Lab Med. 2017;8:52-59.

9. Hersh AR, Megli CJ, Caughey AB. Repeat screening for syphilis in the third trimester of pregnancy: a cost-effectiveness analysis. Obstet Gynecol. 2018;132:699-706.
10. Albright CM, Emerson JB, Werner EF, et al. Third trimester prenatal syphilis screening: a cost-effectiveness analysis. Obstet Gynecol. 2015;126:479-485.

11. Seña AC, White BL, Sparling PF. Novel Treponema pallidum serologic tests: a paradigm shift in syphilis screening for the 21st century. Clin Infect Dis. 2010;51:700-708.

12. Owusu-Edusei K Jr, Peterman TA, Ballard RC. Serologic testing for syphilis in the United States: a cost-effectiveness analysis of two screening algorithms. Sex Transm Dis. 2011;38:1-7.

13. Huh HJ, Chung JW, Park SY, et al. Comparison of automated treponemal and nontreponemal test algorithms as first-line syphilis screening assays. Ann Lab Med. 2016;36:23-27.

14. Centers for Disease Control and Prevention. Syphilis testing algorithms using treponemal test for initial screening-four laboratories. New York City, 2005-2006. MMWR Morb Mortal Wkly Rep. 2008;57:872-875.

15. Mishra S, Boily MC, Ng V, et al. The laboratory impact of changing syphilis screening from the rapid-plasma reagin to a treponemal enzyme immunoassay: a case-study from the greater Toronto area. Sex Transm Dis. 2011;38:190-196.

16. Klein VR, Cox SM, Mitchell MD, et al. The Jarisch-Herzheimer reaction complicating syphilotherapy in pregnancy. Obstet Gynecol. 1990;75:375-380. 